> tip of the iceberg", says Sprenger. There is a major risk that the disease could become endemic in Syria, adds Rosenbauer.

Israel faces a different but also concerning situation. It has high levels of child immunization against polio, but wild poliovirus has been found in sewage in several towns in southern Israel since February. The virus has also been detected in the West Bank and Gaza. The ECDC and the WHO estimate a high risk of international spread of poliovirus from Israel, given the prolonged circulation of virus over a large area.

Israel has so far identified 42 people shedding poliovirus in their faeces. None of them had symptoms of paralysis, and they had been fully vaccinated with inactivated poliovirus vaccine (IPV), which is used in routine immunizations and protects against all polio strains. This is the first time that widespread wild polio has been found without any clinical cases. Most EU countries use IPV, and if exposed to imported polio they could be faced with silent spread of the virus in the environment too, putting unvaccinated populations, particularly infants, at risk. IPV gives a high level of individual protection, but provides poor gut immunity, meaning that vaccinated people might still shed the virus in

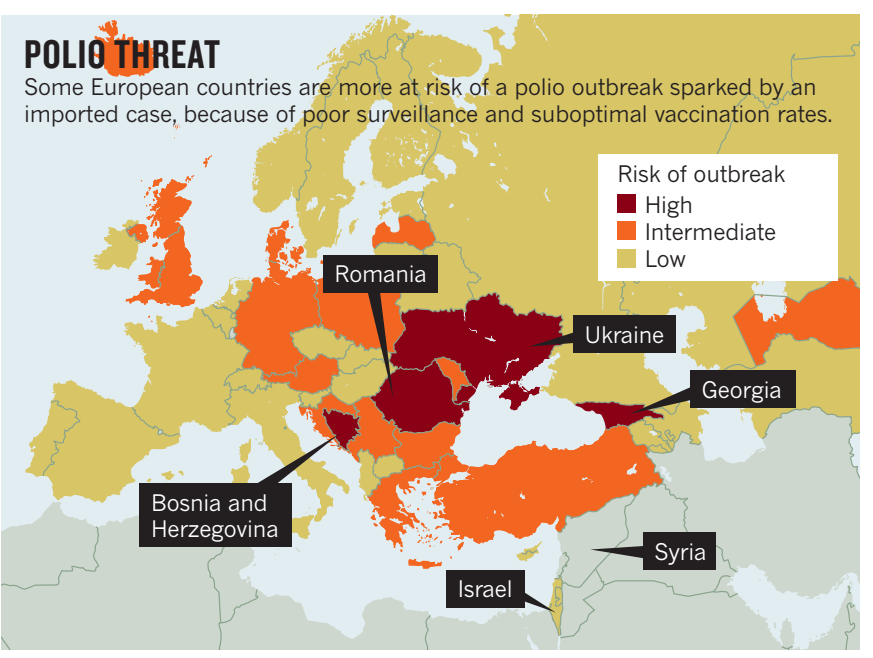

planning a vaccination campaign in all neighbouring countries. The appearance of polio "is going to have implications beyond Syria”, says ơ Rosenbauer.

Israel's effective sewage-surveillance systems were able to detect the virus before any clinical cases occurred, but in Europe, only a handful of countries monitor sewage. Surveillance for acute flaccid paralysis is also often poor ${ }^{2,3}$. The risk that imported cases could go undetected and spread before causing outbreaks is very real, says Sprenger. Europeans who are vaccinated would be protected. But in many countries, including Ukraine, Romania and even some

faeces. An alternative is oral poliovirus vaccine (OPV), a weakened form of the live virus that provides strong gut immunity and prevents faecal shedding. It is used for mass vaccinations and outbreak control because it is effective, cheap and easy to administer. But in rare cases it can cause polio, so polio-free countries prefer to use IPV, which carries no such risk.

To stop silent transmission, Israel has since August given OPV to more than 890,000 children, and Syria has begun administering OPV to 2.4 million children. The WHO and the United Nations Children's Fund (Unicef) are richer nations, polio vaccination rates can be suboptimal. Up to 12 million EU children are not vaccinated against polio.

"We need to improve environmental surveillance and not wait until we have a clinical case of polio," says Sprenger.

1. ECDC. Suspected outbreak of poliomyelitis in Syria: Risk of importation and spread of poliovirus in the EU (ECDC, 2013).

2. ECDC. Wild-type poliovirus 1 in Israel - what is the risk to the EU/EEA? (ECDC, 2013)

3. WHO. Report of the 27th Meeting of the European Regional Certification Commission for Poliomyelitis Eradication (WHO, 2013)

\title{
Root of maths genius sought
}

\section{Entrepreneur's 'Project Einstein' taps 400 top academics for their DNA.}

\section{BY ERIKA CHECK HAYDEN}

$\mathrm{H}$ e founded two genetic-sequencing companies and sold them for hundreds of millions of dollars. He helped to sequence the genomes of a Neanderthal man and James Watson, who co-discovered DNA's double helix. Now, entrepreneur Jonathan Rothberg has set his sights on another milestone: finding the genes that underlie mathematical genius.

Rothberg and physicist Max Tegmark, who is based at the Massachusetts Institute of Technology in Cambridge, have enrolled about 400 mathematicians and theoretical physicists from top-ranked US universities in a study dubbed 'Project Einstein'. They plan to sequence the participants' genomes using the Ion Torrent machine that Rothberg developed.

The team will be wading into a field fraught with controversy. Critics have assailed similar projects, such as one at the BGI (formerly the Beijing Genomics Institute) in Shenzhen, China, that is sequencing the genomes of 1,600 people identified as mathematically precocious children in the 1970s (see Nature 497, 297-299; 2013). The critics say that the sizes of these studies are too small to yield meaningful results for such complex traits. And some are concerned about ethical issues. If the projects find genetic markers for maths ability, these could be used as a basis for the selective abortion of fetuses or in choosing between embryos created through in vitro fertilization, says Curtis McMullen. A mathematician at Harvard University in Cambridge, Massachusetts, and a 1998 winner of the prestigious Fields Medal, McMullen was asked to participate in Project Einstein and declined.

Rothberg is pushing ahead. "I'm not at all concerned about the critics," he says, adding that he does not think such rare genetic traits could be useful in selecting for smarter babies. Influenced by a college class he took from a pioneer in artificial intelligence, and by the diagnosis of his daughter with tuberous sclerosis complex, a disease that can cause mental retardation and autism, Rothberg has long been interested in cognition. He is also in awe of the abilities of famous scientists. "Einstein said 'the most incomprehensible thing about the Universe is that it is comprehensible," he says. "I'd love to find the genes that make the Universe comprehensible."

There is precedent to the concept of sequencing extreme outliers in a population in the hunt for influential genes. Scientists have used the technique to sift for genes that influence medical conditions such as high blood pressure and bone loss. Some behavioural geneticists, such as Robert Plomin at King's College London, who is involved with the BGI project, say that there is no reason that this same approach won't work for maths ability. As much as twothirds of a child's mathematical aptitude seems to be influenced by genes (Y. Kovas et al. Psychol. Sci. 24, 2048-2056; 2013). 
But other geneticists say that intelligence is so complex a trait, influenced by so many genes, that not even a sample of thousands of people would yield sufficient statistical power to get at its genetic basis. They point to studies such as one published in May that examined the genomes of more than 125,000 people and found only three genetic markers with a small effect on how long an individual stays at school (C. A. Rietveld et al. Science 340, 1467-1471; 2013). Project Einstein "is unlikely to have any statistical power", says geneticist Daniel MacArthur at Massachusetts General Hospital in Boston who, with colleagues, has amassed a pedigree of 13 million related people to try to tackle the heritability of complex traits (see Nature http://doi.org/ppj; 2013).

Some participants in Project Einstein are intrigued by the chance to learn about their own genetic sequences, which will be shared with them. "As a science-fiction fan, I like the idea of having my own genome sequenced," says David Aldous, a mathematician at the University of California, Berkeley. "Maybe I'll print a segment onto a T-shirt."

Others say they wouldn't be surprised if the study found that maths aptitude was not born so much as made. "I feel that the notion of 'talent' may be overrated," says Michael Hutchings, a mathematician also at Berkeley. He adds that even if genetic markers are found, they could be used for good - not to pre-select for maths geniuses, but to help parents to understand the particular abilities of their children and give them the support they need.

However, McMullen is concerned that the project is appealing to participants' self-interest without disclosing enough information. "I thought it was strange that it was called 'Project Einstein', which seemed designed to appeal to the participants' egos," he says. He asked the project's staff and the New England Institutional Review Board, which approved the study, to explain how results would be used. "The uniform answer to my questions was that 'we are not responsible for how the information is used after the study is completed"' he says.

The Rothberg Institute for Childhood Diseases, Rothberg's private foundation based in Guilford, Connecticut, is the study's sponsor. But Rothberg won't say who is funding the project, which other geneticists estimate will cost at least US\$1 million. Some speculate that Rothberg is funding it himself. In 2001, Fortune estimated his net worth to be $\$ 168$ million, and that was before he sold the sequencing companies he founded - 454 Life Sciences and Ion Torrent, both based in Connecticut - for a combined total of $\$ 880$ million.

Rothberg is adamant that the project is well worth the time and the money, whoever is paying for it. "This study may not work at all," he says - before adding, quickly, that it "is not a crazy thing to do". For a multimillionaire with time on his hands, that seems to be justification enough.

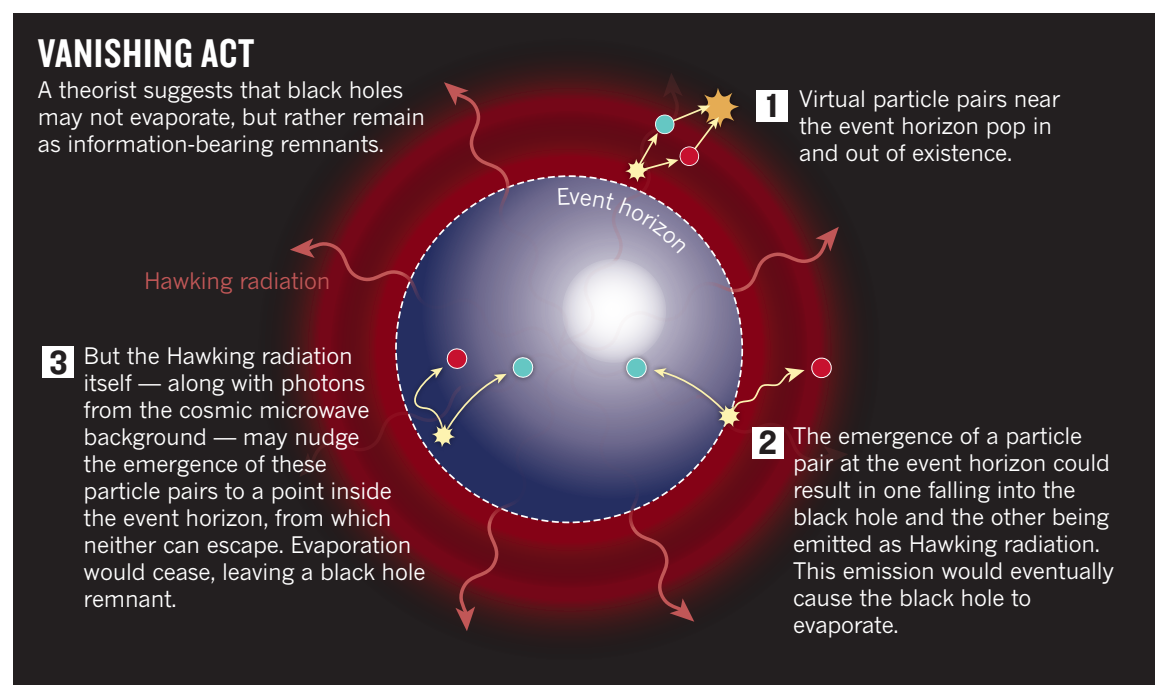

ASTROPHYSICS

\section{Black holes shrink but endure}

\section{Theorist's idea takes on information-preservation problem.}

\section{BY RON COWEN}

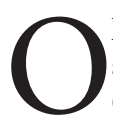
ld black holes never die, they just fade away. So says veteran cosmologist George Ellis of the University of Cape Town in South Africa, who suggests that the cosmos may be littered with an untold number of shrunken black hole remnants.

Ellis' speculative report, posted on 17 October on the preprint server $\operatorname{arXiv}$ (G. F. R. Ellis http://arxiv.org/abs/1310.4771;2013), seems to undermine the seminal work of Stephen Hawking, a cosmologist at the University of Cambridge, UK. In 1974, Hawking calculated that, owing to quantum effects, black holes are not entirely black: some particles escape the black hole's gravitational barrier, known as the event horizon. For a solar-mass black hole, these particles, known as Hawking radiation, would be emitted over the course of $10^{67}$ years until the object vanished without a trace (S. W. Hawking Nature 248, 30-31; 1974).

Although many physicists are sceptical about Ellis's work, it highlights a long-running debate over the ultimate fate of black holes. Uncertainties abound because of the difficulties in reconciling quantum theory which predicts the Hawking radiation - and Einstein's classical theory of gravitation, which defines a black hole's structure. "This is very much a living issue that people are confused about," says Vijay Balasubramanian, a string theorist at the University of
Pennsylvania in Philadelphia.

The debate also touches on one of the most cherished beliefs about the Universe: that information is always preserved. If black holes evaporate, then the information they contain may die along with them. By contrast, a black hole remnant would offer a way in which information might be preserved (even if it could never be extracted). By serving as a storehouse, a remnant "could be a fantastic way of resolving all the issues we have with black holes", says theoretical physicist Jeff Murugan of the University of Cape Town.

In Hawking's original view, quantum theory permits large fluctuations in energy for brief moments of time. As a consequence, the vacuum of space seethes with particle-antiparticle pairs that continually pop in and out of existence (see 'Vanishing act'). When this occurs at the event horizon, one member of a particle pair could be sucked into the black hole, whereas the other could escape as Hawking radiation, depleting the black hole's mass.

But Ellis says that this radiation has another effect. According to Einstein, any source of mass or energy distorts space. A black hole, a body so massive that space closes in on itself, is an extreme example of that distortion. The Hawking radiation would add even more distortion, Ellis says, and so, too, would the ubiquitous photons from the cosmic microwave background, the bath of radiation left over from the Big Bang. He says that these two 